\title{
THE DYNAMIC INTERACTION OF TWIN TUNNELS EMBEDDED IN A HOMOGENEOUS HALF-SPACE
}

\section{Waleed I. Hamad ${ }^{1}$, Hugh E.M. Hunt ${ }^{1}$, James P. Talbot ${ }^{1}$, Mohammed F.M. Hussein ${ }^{2}$, and David J. Thompson ${ }^{3}$}

\author{
${ }^{1}$ Department of Engineering, University of Cambridge \\ Trumpington Street, Cambridge, CB2 1PZ, UK \\ e-mail: $\{$ wih22, hemh1,jpt1000\}@cam.ac.uk \\ ${ }^{2}$ Department of Civil and Architectural Engineering, College of Engineering, Qatar University \\ Doha, 2713, Qatar \\ e-mail:mhussein@qu.edu.qa \\ ${ }^{3}$ Institute of Sound and Vibration Research, University of Southampton \\ Highfield, Southampton, SO17 1BJ, UK \\ e-mail:djt@isvr.soton.ac.uk
}

Keywords: Railway, Ground-borne Vibration, Soil-Structure Interaction, Twin Tunnels.

\begin{abstract}
Vibration generated by underground railways can be a major source of disturbance to occupants of nearby buildings. The numerical prediction of ground-borne vibration is a complicated problem that has received extensive research attention in the past decades. However, simplifying assumptions are always required in state-of-the-art numerical models in order to reduce computational effort and resources. A common simplifying assumption is to neglect the presence of a neighbouring tunnel, despite the fact that many underground railway lines around the world consist of two tunnels. This paper investigates the dynamic interaction of two parallel tunnels embedded in a homogeneous half-space. Two different methods are used to tackle the problem. The first is a fully coupled approach, where two cavities are generated in the halfspace to which the tunnels are coupled. The second adopts a superposition approach, where two sub-models each consisting of a single tunnel are superposed to calculate the vibration response of the twin tunnels. In both modelling approaches, the tunnel wall is modelled using the thick-shell theory, while the boundary element method is used to simulate the half-space of the ground. The vibration response of the twin-tunnel system on the ground surface due to a harmonic point load at one tunnel invert is studied and compared to that of a single tunnel. The efficacy of the superposition method in modelling the dynamic interaction of the twin tunnels is assessed against the fully coupled approach at different points on the surface. The results reveal the significance of the interaction between the twin tunnels and also demonstrate the accuracy of the superposition method. The feasibility of the superposition method in modelling the dynamic interaction between tunnels and piled-foundations is also highlighted.
\end{abstract}




\section{INTRODUCTION}

Underground railway systems are an environmentally-friendly solution to society's increasing demand for mass transport. A growing issue associated with railways, nevertheless, is ground-borne noise and vibration that can cause significant annoyance within the built environment. Vibration is generated at the wheel-rail interface, due to wheel and track irregularities, and propagates to buildings in close proximity with dominant frequencies in the range from $25 \mathrm{~Hz}$ to $250 \mathrm{~Hz}$. Within this frequency range, both ground-borne vibration and re-radiated noise may be perceived, causing discomfort to people and malfunctioning of sensitive equipments.

In the past decades, the numerical prediction of ground-borne vibration from railways has received extensive research effort. Several numerical models have been developed for scrutinising the propagation of vibration from underground railway tunnels adopting, for example, semi-analytical [1, 2, 3], finite-infinite element [4] and coupled finite element-boundary element (FE-BE) [3, 5, 6, 7] approaches. The required number of parameters, however, to accurately simulate the underground environment makes the formulation of a comprehensive model a challenging task, due mainly to computational resources. For this reason, most researchers have adopted simplifying assumptions in the modelling process such as assuming homogeneous or horizontally layered soil, imposing perfect contact at the soil-structure interface and neglecting the existence of nearby buildings. Recently, some attention have been paid to the influence of these simplifying assumptions, such as inclined layers and inhomogeneities of soil [8, 9, 10], voids at the tunnel-soil interface [11] and source-building interaction [12, 13].

Another common simplifying assumption adopted in the majority of numerical studies of vibration from underground railway tunnels is to neglect the presence of a neighbouring tunnel. Twin tunnels constitute many underground railway lines around the world, such as in London, Bangkok and Washington, DC, where one tunnel is used for the outbound direction and the other is used for the inbound direction [14]. There exist in the literature a number of investigations studying the static effects of excavating twin tunnels. However, with regard to the dynamic interaction of twin tunnels, to date only two models are available in the literature. The first is a wavenumber FE-BE model of twin tunnels embedded in a half-space developed by Sheng et al. [15]. This model compares the performance of twin tunnels with that of a single double-track tunnel in terms of vibration response on the ground surface. The second model is a semianalytical model of twin tunnels buried in a full-space developed by Kuo et al. [14]. In this model, which is based on the well-known PiP model [1, 2], the dynamic interaction of twin tunnels is acquired from the solution of a single tunnel model.

This paper presents a model of two parallel tunnels embedded in a homogeneous half-space, in order to investigate the effect of a neighbouring tunnel on the propagation of surface groundborne vibration. Two different methods are used to tackle the problem. The first is a fully coupled approach, where two cavities are generated in the half-space soil to which the tunnels are coupled. The second is a superposition method, where two sub-models, each consisting of a single tunnel, are superposed together to calculate the vibration response of the twin tunnels. In both modelling approaches, the tunnels are modelled using the cylindrical thick-shell theory, while the half-space soil is simulated using the BE method. The system is assumed invariant in the longitudinal direction allowing for the formulation of the governing equations in the wavenumber-frequency domain and hence implementing a $2.5 \mathrm{D}$ approach.

The paper falls into four sections. Section 2 summarises the modelling procedure of the soil and tunnel and describes the superposition method and its governing equations. Section 3 gives the simulation results and comparisons, and finally Section 4 highlights the concluding remarks. 


\section{MODELLING STRATEGY}

The modelling work presented in this paper is aimed at studying the dynamic interaction between twin tunnels in a homogeneous half-space (Fig. 1). Two techniques are adopted: one is a fully coupled approach and the other applies the superposition method. The fully coupled approach is based on generating two cavities in the half-space soil in which the tunnels are inserted, whereas the superposition method produces the response of twin tunnels from the solution of a single tunnel. In both techniques, the soil cavities are modelled using the BE method, while the cylindrical thick-shell theory is used for simulating the tunnel wall. It must be noted that, both the fully coupled approach and superposition method account for the strong coupling between the twin tunnels.

In the analysis, it is assumed that the tunnel is invariant in the longitudinal direction allowing for the use of a $2.5 \mathrm{D}$ approach. In this approach, the calculations are performed in the wavenumber domain $\left(\xi_{y}\right)$, thereby representing the full 3D response of the structure and the radiated wave field on a $2 \mathrm{D}$ mesh. Hence, a frequency response function (FRF) matrix of a 2D cavity is calculated at the frequency of interest and coupled to the FRF matrix of the tunnel wall. The response in the space domain is obtained by applying the inverse Fourier transform as

$$
u(y, \omega)=\frac{1}{2 \pi} \int_{-\infty}^{\infty} u\left(\xi_{y}, \omega\right) e^{\left(i \xi_{y} y\right)} d \xi_{y},
$$

where $u\left(\xi_{y}, \omega\right)$ is the response in the wavenumebr domain. The following sections describe the methods applied in modelling the soil and the tunnel, and present the superposition method.

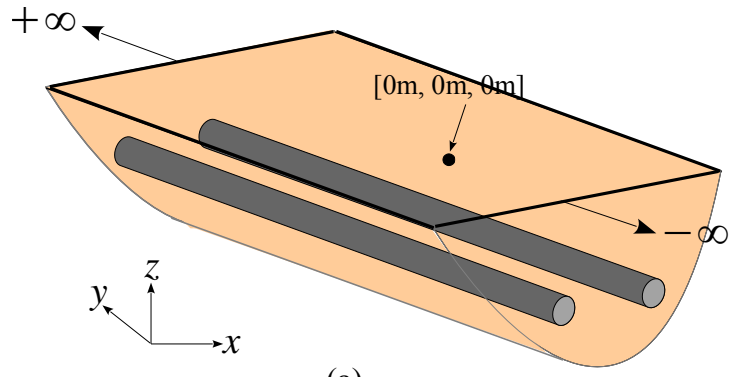

(a)

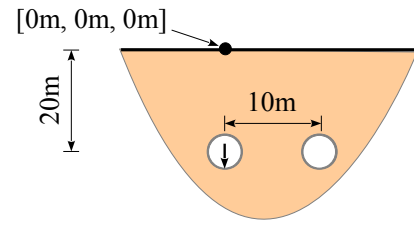

(b)

Figure 1: (a) 3D and (b) 2D (invariant section) illustrations of the layout of the model for twin tunnels embedded in a homogeneous half-space.

\subsection{The soil model}

The soil is modelled using the BE method, based on 2.5D Green's functions for a homogeneous half-space obtained with the aid of the ElastoDynamics Toolbox (EDT) [16]. These fundamental solutions are calculated numerically based on the direct stiffness and thin-layer methods of modelling wave propagation in layered media.

The BE mesh consists of a total of $N_{s}$ constant nodal-collocated elements, at which tractions and displacements are assumed to be uniform. For each of the $N_{s}$ nodes of the BE mesh, there are three values of displacements and tractions related by

$$
\mathbf{H u}=\mathbf{G p}
$$


where $\mathbf{H}$ and $\mathbf{G}$ are $3 N_{s} \times 3 N_{s}$ matrices describing the behaviour of the soil in terms of its Young's modulus $(E)$, density $(\rho)$, Poisson's ratio $(\nu)$, damping ratio $(\eta)$ and frequency of interest $(f)$. The $3 N_{s} \times 1$ vectors $\mathbf{u}$ and $\mathbf{p}$ are assembled from the complex displacement and traction amplitudes of each node as

$$
\begin{aligned}
& \mathbf{u}=\left\{u_{x}^{1} u_{y}^{1} u_{z}^{1} u_{x}^{2} u_{y}^{2} u_{z}^{2} \cdots u_{x}^{N_{s}} u_{y}^{N_{s}} u_{z}^{N_{s}}\right\}^{\top} \\
& \mathbf{p}=\left\{p_{x}^{1} p_{y}^{1} p_{z}^{1} p_{x}^{2} p_{y}^{2} p_{z}^{2} \cdots p_{x}^{N_{s}} p_{y}^{N_{s}} p_{z}^{N_{s}}\right\}^{\top}
\end{aligned}
$$

where $\mathbf{u}^{j}$ and $\mathbf{p}^{j}$ are the displacement and traction vectors of node $j$.

Eq. (2) is rearranged as

$$
\begin{aligned}
\mathbf{u} & =\mathbf{H}^{-1} \mathbf{G p} \\
\mathbf{u} & =\mathbf{H}_{s} \mathbf{p}
\end{aligned}
$$

in which $\mathbf{H}_{s}$ is the FRF matrix relating displacements and tractions at the frequency of interest. Throughout the BE analysis, a minimum of six constant elements are used per wavelength to satisfy the Domínguez recommendations [17] and achieve convergence.

\subsection{The tunnel model}

The tunnel is simulated by the cylindrical thick-shell theory, in which the equations of motion are formulated in the wavenumber-frequency domain. The tunnel is assumed periodic around the tangential coordinate $(\theta)$, and hence any applied force can be represented by a Fourier series of sine and cosine components. If the applied loading comprises harmonic components in space and time, the equations of motion are satisfied by similarly harmonic displacement components. Thus, the modal displacements $\left\{\tilde{U}_{r n}, \tilde{U}_{\theta n}, \tilde{U}_{z n}\right\}^{\top}$ at the mean radius $(a)$ of the cylinder due to applied forces in the radial, tangential and longitudinal directions $\left\{\tilde{Q}_{r n}, \tilde{Q}_{\theta n}, \tilde{Q}_{z n}\right\}^{\top}$ read,

$$
\left\{\begin{array}{l}
\tilde{U}_{r n} \\
\tilde{U}_{\theta n} \\
\tilde{U}_{z n}
\end{array}\right\}=[\mathbf{U}]_{r=a}[\mathbf{T}]_{r=r_{i}, r=r_{e}}^{-1}\left\{\begin{array}{l}
\tilde{Q}_{r n} \\
\tilde{Q}_{\theta n} \\
\tilde{Q}_{z n}
\end{array}\right\},
$$

where the radial and tangential displacements are associated with $\cos n \theta$, and the tangential is associated with $\sin n \theta$ for the symmetrical loading and vice versa for the anti-symmetrical loading. The top half of matrix $[\mathbf{T}]$ is calculated by substituting the inner radius and the bottom half is obtained by substituting the outer radius. The elements of matrices $[\mathbf{U}]$ and $[\mathbf{T}]$ can be found in [14] for symmetric and antisymmetric cases.

Since the tunnel in this paper is subject to a point harmonic load at its invert, it is necessary to decompose the point load into its space-harmonic components before finding the corresponding displacement. The variation of the load can be written as a linear combination of the ring modes $(n)$ by means of a Fourier series as,

$$
\frac{\delta(\theta)}{a}=\frac{1}{2 \pi a}+\sum_{n=1}^{\infty} \frac{1}{\pi a} \cos n \theta=\sum_{n=1}^{\infty} b_{n} \cos n \theta,
$$

on the interval $-\pi<\theta \leq \pi$, where $\delta(\theta)$ is the Dirac delta function. Using the representation of the point load in Eq. (6), the input force can be written in a form that suits the formulation in Eq. (5) in order to calculate the response due to symmetric and antisymmetric forces for a given ring mode $(n)$.

The FRF matrix of the tunnel is formulated using the dynamic stiffness approach by dividing the shell into $N_{t}$ nodes. Each node has three degrees-of-freedom (DoFs) representing the longitudinal, tangential and radial directions (Fig. 2). With the aid of the thick-shell formulation 
in Eq. (5) and the representation of the point load in Eq. (6), a FRF matrix $\left(\mathbf{H}_{t}\right)$ relating the response at each DoF to the applied force is assembled.

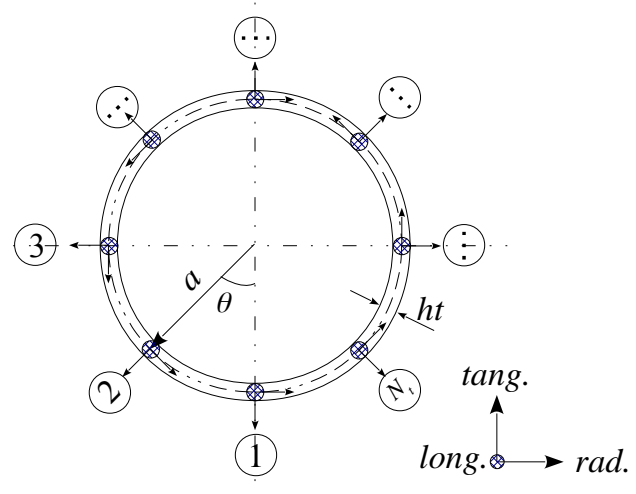

Figure 2: Cross-section a thick-shell divided into $N_{t}$ nodes.

Before combining, the systems in Eqs. (2) and (5), two transformations are carried out. First, the soil FRF matrix is modified to relate displacements and forces instead of tractions by dividing it by the area of the elements in the BE mesh. Second, the tunnel FRF matrix is modified to relate displacements and forces in the Cartesian coordinates rather than in the cylindrical coordinates as,

$$
\mathbf{H}_{t}^{c}=\mathbf{T}_{r}^{\top} \mathbf{H}_{t} \mathbf{T}_{r}
$$

where the size of the transformation matrix $\mathbf{T}_{r}$ is $3 N_{t} \times 3 N_{t}$.

The tunnel can now be coupled to the soil cavity in the wavenumber domain by applying compatibility of displacements, i.e. soil displacement $\left(\mathbf{U}_{s}\right)$ equals tunnel displacement $\left(\mathbf{U}_{t}\right)$ and equilibrium of forces at the interface as

$$
\begin{aligned}
& \mathbf{U}_{s}=\mathbf{H}_{s} \mathbf{F}_{s} \\
& \mathbf{U}_{t}=\mathbf{H}_{t}^{c}\left(\mathbf{F}_{a}-\mathbf{F}_{s}\right) \\
& \mathbf{F}_{s}=\left(\mathbf{H}_{s}+\mathbf{H}_{t}^{c}\right)^{-1} \mathbf{H}_{t}^{c} \mathbf{F}_{a}
\end{aligned}
$$

in which $\mathbf{F}_{a}$ is the force applied in the tunnel and $\mathbf{F}_{s}$ is the resulting force applied in the soil. Knowing $\mathbf{F}_{s}$, the response at any point in the soil can be calculated.

\subsection{The superposition method}

The superposition method is used to solve the system in Fig. 1 by breaking it into two submodels, each representing a single cavity embedded in a homogeneous half-space. As shown in Fig. 3, each sub-model has a horizontal cavity, referred to here as a real cavity, and a virtual cavity at the position of the real cavity in the other sub-model. The method used in this paper is similar to that applied by Kuo et al. [14] for modelling twin tunnels embedded in a full-space.

The displacement fields for each sub-model due to forces acting on a single cavity can be calculated at its interface and also at the interface of the virtual cavity. The vibration response of the coupled system can be written as the superposition of the two displacement fields $\left(\mathbf{U}^{r}, \mathbf{U}^{v}\right)$. One displacement field is the result of forces acting on a single cavity, while the other is the result of the interaction between the two cavities. Likewise, the total forces applied to the 


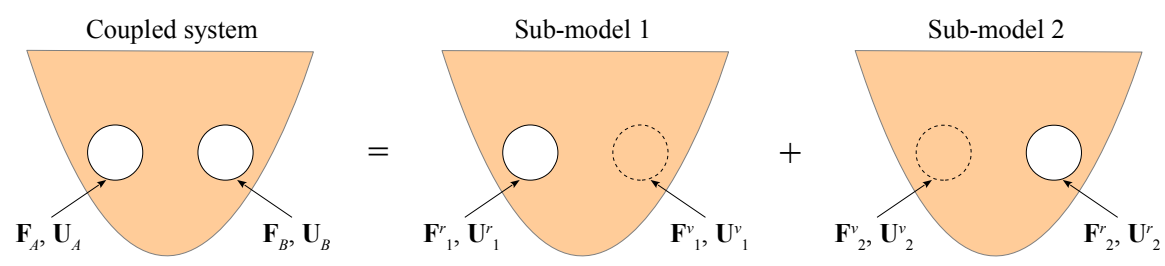

Figure 3: Schematic for the superposition method used for solving the twin tunnels, showing the coupled system and the sub-models. The subscripts $A$ and $B$ refer to the cavity number, and 1 and 2 denote sub-model 1 respectively sub-model 2 , whereas the superscripts $r$ and $v$ refer to real and virtual cavity respectively.

coupled system $\left(\mathbf{F}^{r}, \mathbf{F}^{v}\right)$ are equal to the summation of the forces acting on one cavity with those representing the motion induced by the neighbouring cavity. This is described by the following equations,

$$
\begin{aligned}
\mathbf{U}_{A} & =\mathbf{U}_{1}^{r}+\mathbf{U}_{2}^{v} \\
\mathbf{F}_{A} & =\mathbf{F}_{1}^{r}+\mathbf{F}_{2}^{v} \\
\mathbf{U}_{B} & =\mathbf{U}_{1}^{v}+\mathbf{U}_{2}^{r}, \\
\mathbf{F}_{B} & =\mathbf{F}_{1}^{v}+\mathbf{F}_{2}^{r}
\end{aligned}
$$

in which the subscripts $A$ and $B$ refer to the cavity number and 1 and 2 refer to the sub-model number, and superscripts $r$ and $v$ denote the real and virtual cavity respectively.

From the sub-model 1 in Fig. 3, relationships between the forces $\left(\mathbf{F}_{1}^{r}\right)$ on the real cavity and its displacements $\left(\mathbf{U}_{1}^{r}\right)$ as well as the displacements $\left(\mathbf{U}_{1}^{v}\right)$ and tractions $\left(\mathbf{Q}_{1}^{v}\right)$ on the virtual cavity read

$$
\begin{aligned}
\mathbf{U}_{1}^{r} & =\mathbf{H}_{1}^{r r} \mathbf{F}_{1}^{r} \\
\mathbf{U}_{1}^{v} & =\mathbf{H}_{1}^{v r} \mathbf{F}_{1}^{r}, \\
\mathbf{Q}_{1}^{v} & =\mathbf{G}_{1}^{v r} \mathbf{F}_{1}^{r},
\end{aligned}
$$

where $\mathbf{H}_{1}^{r r}$ is the FRF matrix relating the real cavity forces and displacements, and $\mathbf{H}_{1}^{v r}$ and $\mathbf{G}_{1}^{v r}$ are the FRF matrices relating the real cavity forces and the virtual cavity displacements and forces respectively. The force vector $\left(\mathbf{F}_{1}^{v}\right)$ is obtained by integrating the traction vector $\left(\mathbf{Q}_{1}^{v}\right)$.

Similarly for sub-model 2 in Fig. 3, relationships between the forces on the interface of the real cavity $\left(\mathbf{F}_{2}^{r}\right)$ and its displacements $\left(\mathbf{U}_{2}^{r}\right)$ in addition to the virtual cavity displacements $\left(\mathbf{U}_{2}^{v}\right)$ and tractions $\left(\mathbf{Q}_{2}^{v}\right)$ yield

$$
\begin{aligned}
\mathbf{U}_{2}^{r} & =\mathbf{H}_{2}^{r r} \mathbf{F}_{2}^{r} \\
\mathbf{U}_{2}^{v} & =\mathbf{H}_{2}^{v r} \mathbf{F}_{2}^{r}, \\
\mathbf{Q}_{2}^{v} & =\mathbf{G}_{2}^{v r} \mathbf{F}_{2}^{r},
\end{aligned}
$$

in which $\mathbf{H}_{2}^{r r}$ is the FRF matrix relating the real cavity forces and displacements, and $\mathbf{H}_{2}^{v r}$ and $\mathbf{G}_{2}^{v r}$ are the FRF matrices relating the real cavity forces and the virtual cavity displacements and forces respectively. The force vector $\left(\mathbf{F}_{2}^{v}\right)$ is obtained by integrating the traction vector $\left(\mathbf{Q}_{2}^{v}\right)$.

The FRF matrices $\left(\mathbf{H}_{1}^{r r}, \mathbf{H}_{1}^{v r}, \mathbf{H}_{2}^{r r}\right.$, and $\left.\mathbf{H}_{2}^{v r}\right)$ are obtained by applying a unit force at each DoF of the real cavity $\left(\mathbf{F}_{1}^{r}, \mathbf{F}_{2}^{r}\right)$ and calculating the corresponding displacements of the real cavity $\left(\mathbf{U}_{1}^{r}, \mathbf{U}_{2}^{r}\right)$ as well as the virtual cavity $\left(\mathbf{U}_{1}^{v}, \mathbf{U}_{2}^{v}\right)$. The FRF matrices $\left(\mathbf{G}_{1}^{v r}, \mathbf{G}_{2}^{v r}\right)$ are acquired from the displacements of the virtual cavity by calculating: (i) strains by differentiating displacements, (ii) stresses from strains using constitutive relationships, and (iii) tractions using Caushy's stress law as

$$
\mathbf{Q}=\boldsymbol{\sigma} \mathbf{n}
$$


where $\mathbf{Q}$ is the traction vector, $\boldsymbol{\sigma}$ is the stress tensor and $\mathbf{n}$ is the normal vector.

Now, there are $10 \times 3 N_{s}$ system of equations presented in Eqs. (9) to (11), that can be solved together to give the FRF matrix of the coupled system in Fig. 3. Substituting the displacement vectors of the sub-models in Eqs. (10) and (11) in the displacement vectors of the coupled system in Eq. (9) yields

$$
\left[\begin{array}{l}
\mathbf{U}_{A} \\
\mathbf{U}_{B}
\end{array}\right]=\left[\begin{array}{l}
\mathbf{U}_{1}^{r} \\
\mathbf{U}_{1}^{v}
\end{array}\right]+\left[\begin{array}{l}
\mathbf{U}_{2}^{v} \\
\mathbf{U}_{2}^{r}
\end{array}\right]=\left[\begin{array}{ll}
\mathbf{H}_{1}^{r r} & \mathbf{H}_{2}^{v r} \\
\mathbf{H}_{1}^{v r} & \mathbf{H}_{2}^{r r}
\end{array}\right]\left[\begin{array}{l}
\mathbf{F}_{1}^{r} \\
\mathbf{F}_{2}^{r}
\end{array}\right]
$$

Likewise, substituting the force vectors of the sub-models in Eqs. (10) and (11) in the force vectors of the coupled system in Eq. (9) gives

$$
\left[\begin{array}{c}
\mathbf{F}_{A} \\
\mathbf{F}_{B}
\end{array}\right]=\left[\begin{array}{c}
\mathbf{F}_{1}^{r} \\
\mathbf{F}_{1}^{v}
\end{array}\right]+\left[\begin{array}{c}
\mathbf{F}_{2}^{v} \\
\mathbf{F}_{2}^{r}
\end{array}\right]=\left[\begin{array}{c}
\mathbf{I}_{1} \\
\mathbf{G}_{1}^{v r}
\end{array}\right] \mathbf{F}_{1}^{r}+\left[\begin{array}{c}
\mathbf{G}_{2}^{v r} \\
\mathbf{I}_{2}
\end{array}\right] \mathbf{F}_{2}^{r}=\left[\begin{array}{c}
\mathbf{G}_{2}^{v r} \\
\mathbf{I}_{2}
\end{array}\right] \mathbf{F}_{2}^{r}=\left[\begin{array}{cc}
\mathbf{I}_{1} & \mathbf{G}_{2}^{v r} \\
\mathbf{G}_{1}^{v r} & \mathbf{I}_{2}
\end{array}\right]\left[\begin{array}{c}
\mathbf{F}_{1}^{r} \\
\mathbf{F}_{2}^{r}
\end{array}\right],
$$

where $\mathbf{I}_{1}$ and $\mathbf{I}_{2}$ are identity matrices.

Rearranging Eq. (14) and substituting in Eq. (13), the displacement and force vectors of the coupled system are related together as

$$
\left[\begin{array}{c}
\mathbf{U}_{A} \\
\mathbf{U}_{B}
\end{array}\right]=\left[\begin{array}{ll}
\mathbf{H}_{1}^{r r} & \mathbf{H}_{2}^{v r} \\
\mathbf{H}_{1}^{v r} & \mathbf{H}_{2}^{r r}
\end{array}\right]\left[\begin{array}{cc}
\mathbf{I}_{1} & \mathbf{G}_{2}^{v r} \\
\mathbf{G}_{1}^{v r} & \mathbf{I}_{2}
\end{array}\right]^{-1}\left[\begin{array}{c}
\mathbf{F}_{A} \\
\mathbf{F}_{B}
\end{array}\right]
$$

From Eq. (15), the soil FRF matrix of the coupled system $\left(\mathbf{H}_{s}^{c}\right)$ in Fig. 3 reads

$$
\mathbf{H}_{s}^{c}=\left[\begin{array}{ll}
\mathbf{H}_{1}^{r r} & \mathbf{H}_{2}^{v r} \\
\mathbf{H}_{1}^{v r} & \mathbf{H}_{2}^{r r}
\end{array}\right]\left[\begin{array}{cc}
\mathbf{I}_{1} & \mathbf{G}_{2}^{v r} \\
\mathbf{G}_{1}^{v r} & \mathbf{I}_{2}
\end{array}\right]^{-1}
$$

which is to be coupled to the twin tunnels FRF matrix in the same manner of Eq. (8).

\section{SIMULATION PARAMETERS AND RESULTS}

The simulation results presented in this paper are intended to serve two purposes. The first is to scrutinise the effect of a neighbouring tunnel on the vibration field from an existing tunnel. The second is to testify the validity of the superposition method for modelling the dynamic interaction of twin tunnels. These purposes are met by simulating twin tunnels embedded at a depth of $20 \mathrm{~m}$ and separated by a distance of $10 \mathrm{~m}$, see Fig. 1 . One tunnel is subject to a unit harmonic point load at its invert and the responses on the free-surface are calculated in the frequency range from $1 \mathrm{~Hz}$ to $80 \mathrm{~Hz}$, with a resolution of $1 \mathrm{~Hz}$ for the fully coupled approach and $5 \mathrm{~Hz}$ for the superposition method. A coarse frequency resolution is used for the superposition method to reduce computation time. The number of elements in the BE mesh $\left(N_{s}\right)$, which comprises 40 constant elements of equal size, conforms to those in the tunnel model $\left(N_{t}\right)$. Table 1 summarises the parameters used in the simulations.

\subsection{Effects of a neighbouring tunnel}

The significance of the interaction of twin tunnels is presented here by investigating the displacement fields of single and twin tunnels on the free-surface at example frequencies of $30 \mathrm{~Hz}$ and $70 \mathrm{~Hz}$. Waves generated at the tunnel due to a harmonic point load at the invert propagate through the soil and result in Rayleigh waves at the surface of the half-space. The 


\begin{tabular}{ccc}
\hline Parameter & Tunnel & Soil \\
\hline Radius, $a$ & $2.875 \mathrm{~m}$ & $3 \mathrm{~m}$ \\
Thickness, $h$ & $0.25 \mathrm{~m}$ & - \\
Young's modulus, $E$ & $50 \mathrm{GPa}$ & $550 \mathrm{MPa}$ \\
Density, $\rho$ & $2500 \mathrm{~kg} / \mathrm{m}^{3}$ & $2000 \mathrm{~kg} / \mathrm{m}^{3}$ \\
Poisson's ratio, $\nu$ & 0.3 & 0.44 \\
Damping loss factor, $\eta$ & 0.06 & 0.06 \\
Shear wave velocity, $c_{s}$ & - & $309 \mathrm{~m} / \mathrm{s}$ \\
Compression wave velocity, $c_{p}$ & - & $944 \mathrm{~m} / \mathrm{s}$ \\
\hline
\end{tabular}

Table 1: Tunnel and soil parameters used in the simulations.

results presented in this section are produced by the fully coupled approach. The insertion gain (IG) between the two systems is calculated at both frequencies to quantify the effect of a neighbouring tunnel as

$$
\mathrm{IG}=20 \log _{10}\left(\left|\frac{u(y, \omega)_{\text {twin }}}{u(y, \omega)_{\text {single }}}\right|\right) .
$$

Fig. 4 depicts the transverse ( $x$-axis) displacement field generated by single and twin tunnels due to a harmonic point load at the invert. It can be observed for the single tunnel model that, due to symmetry, there are no displacements on the plane $x=0$, while the displacements at other symmetry points are equal in magnitude and opposite in direction. The insertion of a second tunnel at a centre-to-centre distance of $10 \mathrm{~m}$ clearly disrupts this symmetry, causing diffraction of the wavefronts particularly at $70 \mathrm{~Hz}$. The net effect of the second tunnel is a change in the displacement field of up to $\pm 10 \mathrm{~dB}$ at both frequencies, as illustrated in the insertion gain plots.
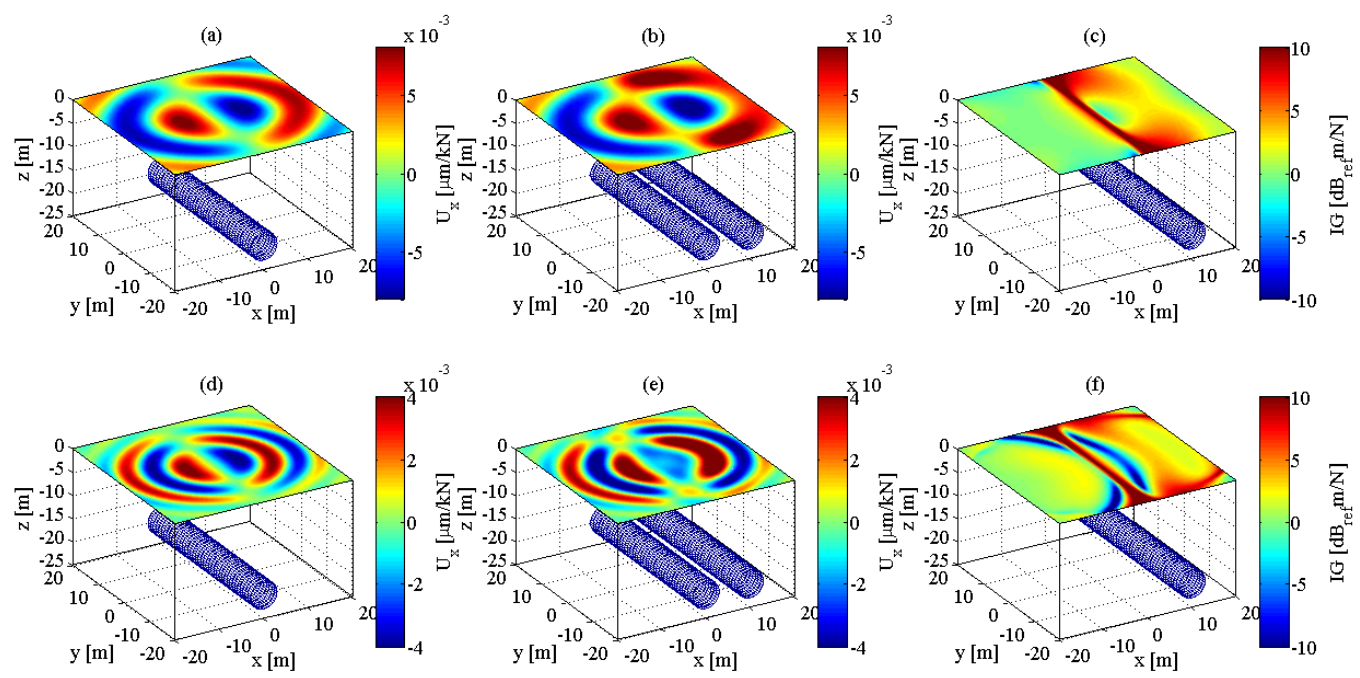

Figure 4: Real part of the transverse ( $x$-axis) displacement on the free-surface due to a unit harmonic point load on one tunnel invert at a frequency of (a-c) $30 \mathrm{~Hz}$ and (d-f) $70 \mathrm{~Hz}$ for (a), (d) a single tunnel and (b), (e) twin tunnels, and (c), (f) the corresponding insertion gain, as computed by the fully coupled approach.

The longitudinal (y-axis) displacement field of the single and twin tunnels due to a harmonic 
point load at the invert is shown in Fig. 5. All displacements on the plane $y=0$ equal zero due to symmetry for both the single and twin tunnels. At other symmetry points, the displacements are equal in magnitude and opposite in direction. Again, the inclusion of a neighbouring tunnel clearly distorts the wavefronts at both frequencies, resulting in an insertion gain of up to $\pm 20 \mathrm{~dB}$.
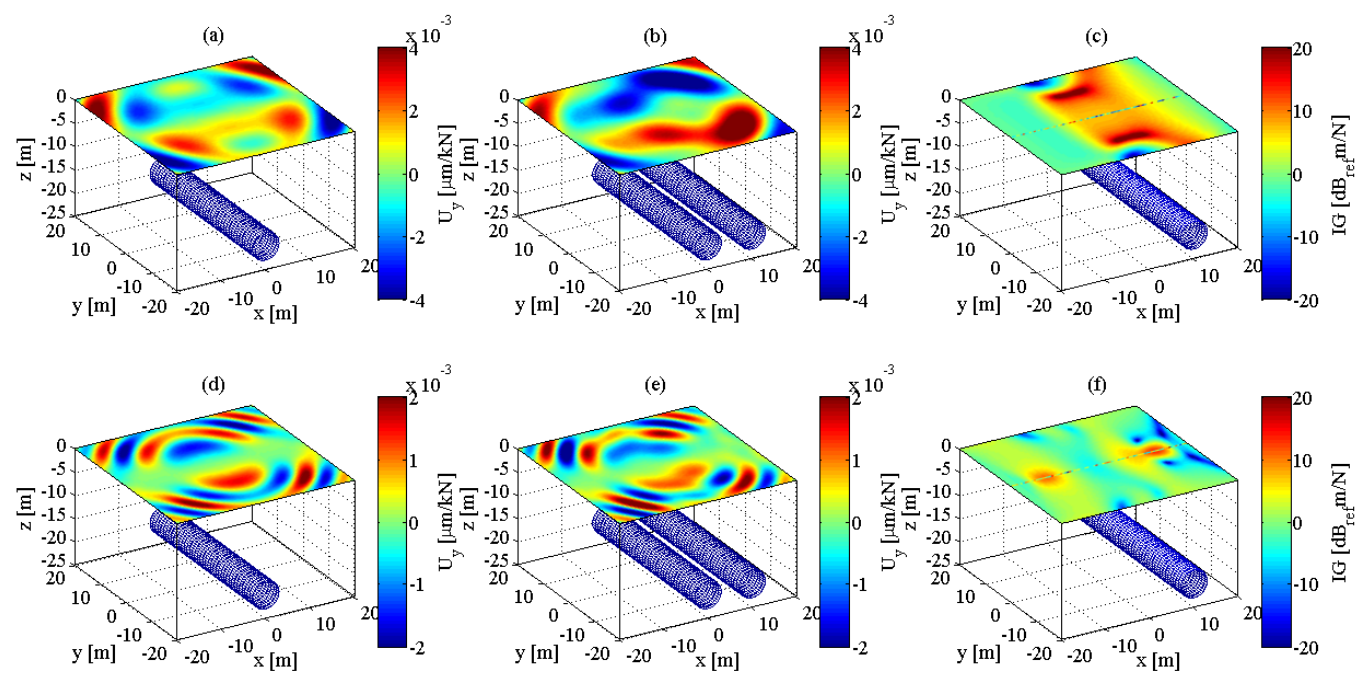

Figure 5: Real part of the longitudinal (y-axis) displacement on the free-surface due to a unit harmonic point load on one tunnel invert at a frequency of (a-c) $30 \mathrm{~Hz}$ and (d-f) $70 \mathrm{~Hz}$ for (a), (d) a single tunnel and (b), (e) twin tunnels, and (c), (f) the corresponding insertion gain, as computed by the fully coupled approach.
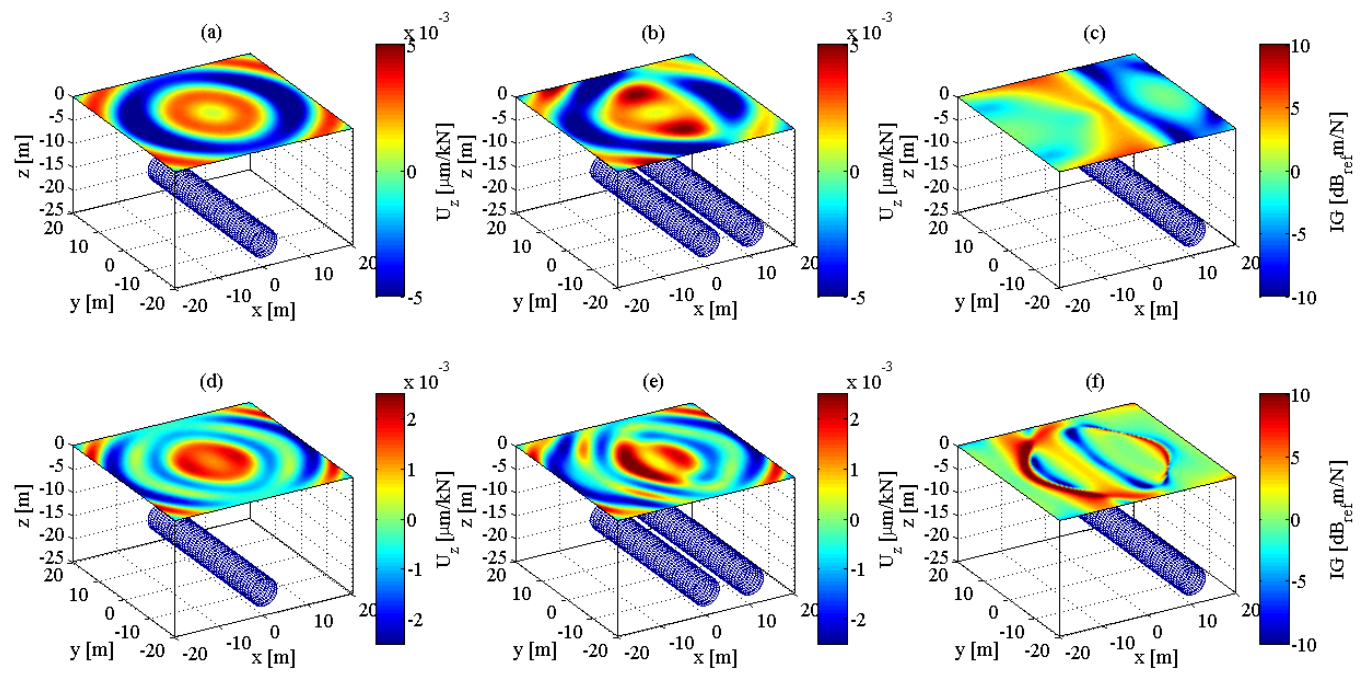

Figure 6: Real part of the vertical (z-axis) displacement on the free-surface due to a unit harmonic point load on one tunnel invert at a frequency of (a-c) $30 \mathrm{~Hz}$ and (d-f) $70 \mathrm{~Hz}$ for (a), (d) a single tunnel and (b), (e) twin tunnels, and (c), (f) the corresponding insertion gain, as computed by the fully coupled approach.

Fig. 6 illustrates the vertical (z-axis) displacement field on the free-surface, which shows that the wavefronts are not cylindrical due to the nature of the source and the dynamic interaction 
between the soil and the tunnels. For the single tunnel, the displacements at the symmetry points are equal, yet they are not equal for the twin tunnels. The addition of the second tunnel modifies the displacement field by up to $\pm 10 \mathrm{~dB}$. It is also noted that the second tunnel appears to diffract the wavefront more than in the horizontal displacement field in Fig. 4.

Similar observations to those presented in Figs. 4 to 6 have been highlighted in a recent study of dynamic interaction between twin tunnels in a full-space [14]. In this study, it was shown that the presence of a neighbouring tunnel can modify the vibration field of an existing tunnel at times of the order of $20 \mathrm{~dB}$.

\subsection{Verification of the superposition method}

The efficacy of the superposition method in modelling the twin tunnels is now assessed against the fully coupled approach. The free-surface vibration response due to a harmonic point load at one tunnel invert is calculated at points $[0 \mathrm{~m}, 0 \mathrm{~m}, 0 \mathrm{~m}],[5 \mathrm{~m}, 0 \mathrm{~m}, 0 \mathrm{~m}],[10 \mathrm{~m}, 0 \mathrm{~m}, 0 \mathrm{~m}]$, $[0 \mathrm{~m}, 10 \mathrm{~m}, 0 \mathrm{~m}],[5 \mathrm{~m}, 10 \mathrm{~m}, 0 \mathrm{~m}]$ and $[10 \mathrm{~m}, 10 \mathrm{~m}, 0 \mathrm{~m}]$ as shown in Fig. 1 . Such predictions might be useful for practising engineers who are often interested in vibration levels on the ground surface for the assessment of train-induced vibration and vibration countermeasures.

Figs. 7 to 9 show the magnitude of the three displacement components $\left(\mathrm{U}_{x}, \mathrm{U}_{y}, \mathrm{U}_{z}\right)$ at the six points on the free-surface as predicted by the fully coupled approach and the superposition method. It is worth noting that the longitudinal displacements at points $[0 \mathrm{~m}, 0 \mathrm{~m}, 0 \mathrm{~m}],[5 \mathrm{~m}$, $0 \mathrm{~m}, 0 \mathrm{~m}]$ and $[10 \mathrm{~m}, 0 \mathrm{~m}, 0 \mathrm{~m}]$ are equal to zero due to symmetry. In general, a good agreement between the two techniques is seen for all displacements, with small differences of less than $0.5 \mathrm{~dB}$ at some frequencies. Such differences are still acceptable for vibration predictions, given their sensitivity to changes in the measurement techniques, environmental conditions and/or material properties.
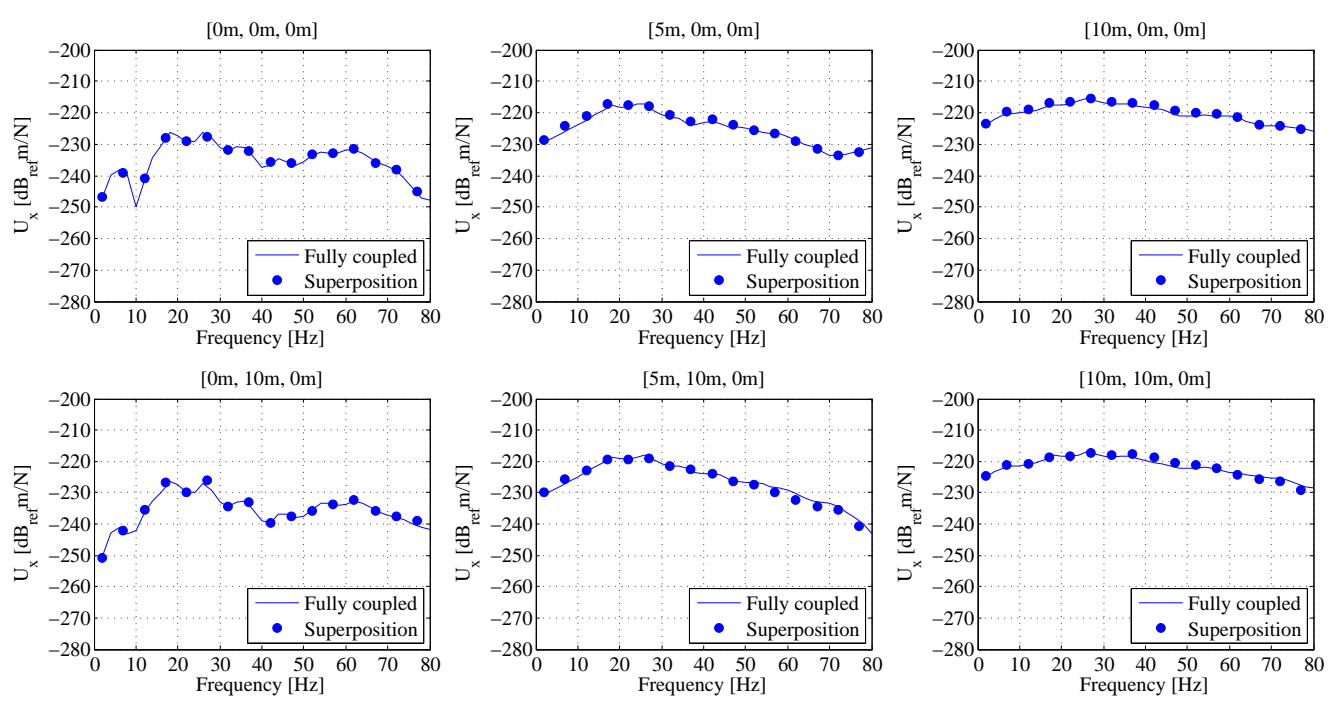

Figure 7: Magnitude of the transverse displacement at points on the free-surface above twin tunnels in a homogeneous half-space due to a unit harmonic point load on one tunnel invert, as computed by the fully coupled and superposition approaches.

Theoretically, the superposition method should give exact results to those of the fully coupled approach since the system in Fig. 1 is linear. The method, however, inevitably involves 
computation errors as it approximates the tractions of the virtual cavity numerically. It is therefore believed that the small differences are due to numerical errors resulting from calculating strains by differentiating displacements.
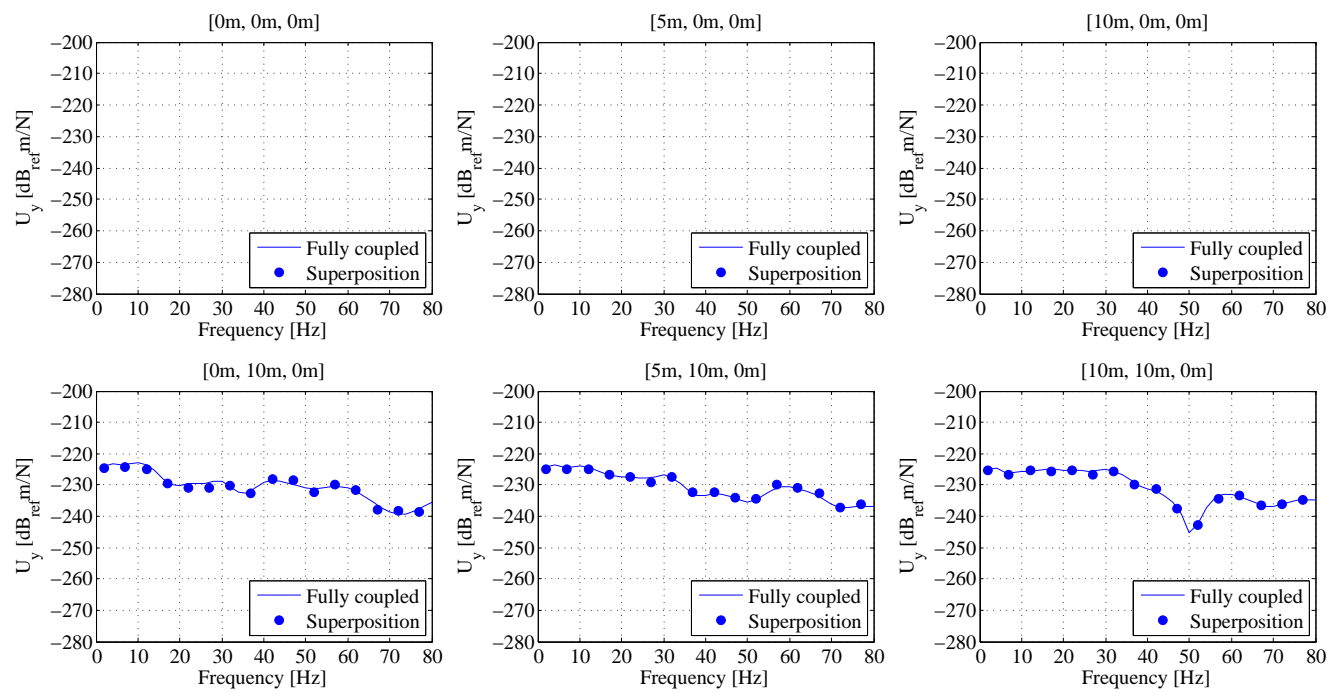

Figure 8: Magnitude of the longitudinal displacement at points on the free-surface above twin tunnels in a homogeneous half-space due to a unit harmonic point load on one tunnel invert, as computed by the fully coupled and superposition approaches.


Figure 9: Magnitude of the vertical displacement at points on the free-surface above twin tunnels in a homogeneous half-space due to a unit harmonic point load on one tunnel invert, as computed by the fully coupled and superposition approaches.

The results shown in Figs. 7 to 9 have indicated that the superposition method can robustly model the dynamic interaction between underground neighbouring structures. This is essential 
for modelling systems that cannot practically be simulated by the fully coupled approach, due to computational limitations, such as those including both fully coupled piled-foundations and underground tunnels. The superposition method is valuable in such cases because it breaks the problem into two manageable sub-models; one representing the tunnels efficiently in the wavenumber domain $(2.5 \mathrm{D})$, and the other representing the full $3 \mathrm{D}$ behaviour of the piledfoundations.

\section{CONCLUSIONS}

There are a number of underground railway lines around the world consisting of twin tunnels, including those in London, Bangkok and Washington, DC. Despite this fact, the majority of previous numerical studies of ground-borne vibration from underground railways consider a single tunnel in the modelling process. This paper has presented two techniques for modelling twin tunnels in a homogeneous half-space, both using the boundary element method for the soil and the cylindrical thick-shell theory for the tunnels. The models have studied the vibration response on the ground surface due to a harmonic point load at one tunnel invert.

It has been demonstrated that the dynamic interaction between twin tunnels is highly significant. At the examined frequencies, the insertion gain of the displacement field on the freesurface between the twin tunnels and the single tunnel reaches up to $\pm 10 \mathrm{~dB}$ for the transverse and vertical directions, and up to $\pm 20 \mathrm{~dB}$ for the longitudinal direction.

The accuracy of the superposition method in predicting the dynamic interaction between twin tunnels has been assessed against the fully coupled approach. Small discrepancies between the two techniques are attributed to the inevitable numerical errors associated with calculating the tractions of the virtual cavity in the superposition method. However, these discrepancies are acceptable, and unlike the fully coupled approach, the superposition method is deemed a feasible technique for modelling the interaction between longitudinally invariant structures and finite structures, such as underground tunnels and piled-foundations.

\section{Acknowledgements}

The authors would like to acknowledge the EPSRC for funding this research, which is part of the MOTIV project (www.motivproject.co.uk), grant numbers (EP/K006665/1, $\mathrm{EP} / \mathrm{K} 006002 / 1, \mathrm{EP} / \mathrm{K} 005847 / 2$ ). We also thank the contribution of the Katholieke Universiteit Leuven for providing the EDT and some functions of BEMFUN used in the BE models.

\section{REFERENCES}

[1] J. A. Forrest, H. E. M. Hunt, A three-dimensional tunnel model for calculation of traininduced ground vibration. Journal of Sound and Vibration, 294, 678-705, 2006.

[2] M. F. M. Hussein, H. E. M. Hunt, A numerical model for calculating vibration from a railway tunnel embedded in a full-space. Journal of Sound and Vibration, 305, 401-431, 2007.

[3] M. F. M. Hussein, S. François, M. Schevenels, H. E. M. Hunt, J. P. Talbot, G. Degrande, The fictitious force method for efficient calculation of vibration from a tunnel embedded in a multi-layered half-space. Journal of Sound and Vibration, 333, 6996-7018, 2014. 
[4] Y. B. Yang, H. H. Hung, A 2.5D finite/infinite element approach for modelling viscoelastic bodies subjected to moving loads. International Journal for Numerical Methods in Engineering, 51, 1317-1336, 2001.

[5] L. Andersen, C. J. C. Jones, Coupled boundary and finite element analysis of vibration from railway tunnels - a comparison of two- and three-dimensional models. Journal of Sound and Vibration, 293, 611-625, 2006.

[6] S. Gupta, M. F. M. Hussein, G. Degrande, H. E. M. Hunt, D. Clouteau, A comparison of two numerical models for the prediction of vibrations from underground railway traffic. Soil Dynamics and Earthquake Engineering, 27, 608-624, 2007.

[7] S. François, M. Schevenels, G. Lombaert, P. Galvín, G. Degrande, A 2.5D coupled FE$\mathrm{BE}$ methodology for the dynamic interaction between longitudinally invariant structures and a layered halfspace. Computer Methods in Applied Mechanics and Engineering, 199 (23-24), 1536-1548, 2010.

[8] S. Jones, H. Hunt, The effect of inclined soil layers on surface vibration from underground railways using the thin layer method. ASCE Journal of Engineering Mechanics, 137 (12), 887-900, 2011.

[9] S. Jones, H. Hunt, Predicting surface vibration from underground railways through inhomogeneous soil. Journal of Sound and Vibration, 331 (9), 2055-2069, 2012.

[10] W. Yang, M. F. M. Hussein, A. M. Marshall, Centrifuge and numerical modelling of ground-borne vibration from an underground tunnel. Soil Dynamics and Earthquake Engineering, 51, 23-34, 2013.

[11] S. Jones, H. Hunt, Voids at the tunnel-soil interface for calculation of ground vibration from underground railways, Journal of Sound and Vibration, 330 (2), 245270, 2011.

[12] P. Coulier, G. Lombaert, G. Degrande, The influence of source-receiver interaction on the numerical prediction of railway induced vibrations. Journal of Sound and Vibration, 333, 2520-2538, 2014.

[13] P. Lopes, P. A. Costa, M. Ferraz, R. Calçada, A. Cardoso, Numerical modelling of vibrations induced by railway traffic in tunnels: From the source to the nearby buildings. Soil Dynamics and Earthquake Engineering, 61-62, 269-285, 2014.

[14] K. Kuo, H. E. M. Hunt, M. F. M. Hussein, The effect of a twin tunnel on the propagation of ground-born vibration from an underground railway. Journal of Sound and Vibration 330, 6203-6222, 2011.

[15] X. Sheng, C. J. C. Jones, D. J. Thompson, Modelling ground vibration from railways using wavenumber finite- and boundary-element methods. Proceedings of the Royal Society A, 461, 2043-2070, 2005.

[16] M. Schevenels, S. François, G. Degrande, EDT: An elastodynamics toolbox for MATLAB. Computers \& Geosciences 35(80), 1752-1754, 2009.

[17] J. Domínguez, Boundary Elements in Dynamics. Computational Mechanics Publications and Elsevier Applied Science, 1993. 